\title{
Characterizing Fault Roughness-Are Faults Rougher at Long or Short Wavelengths?
}

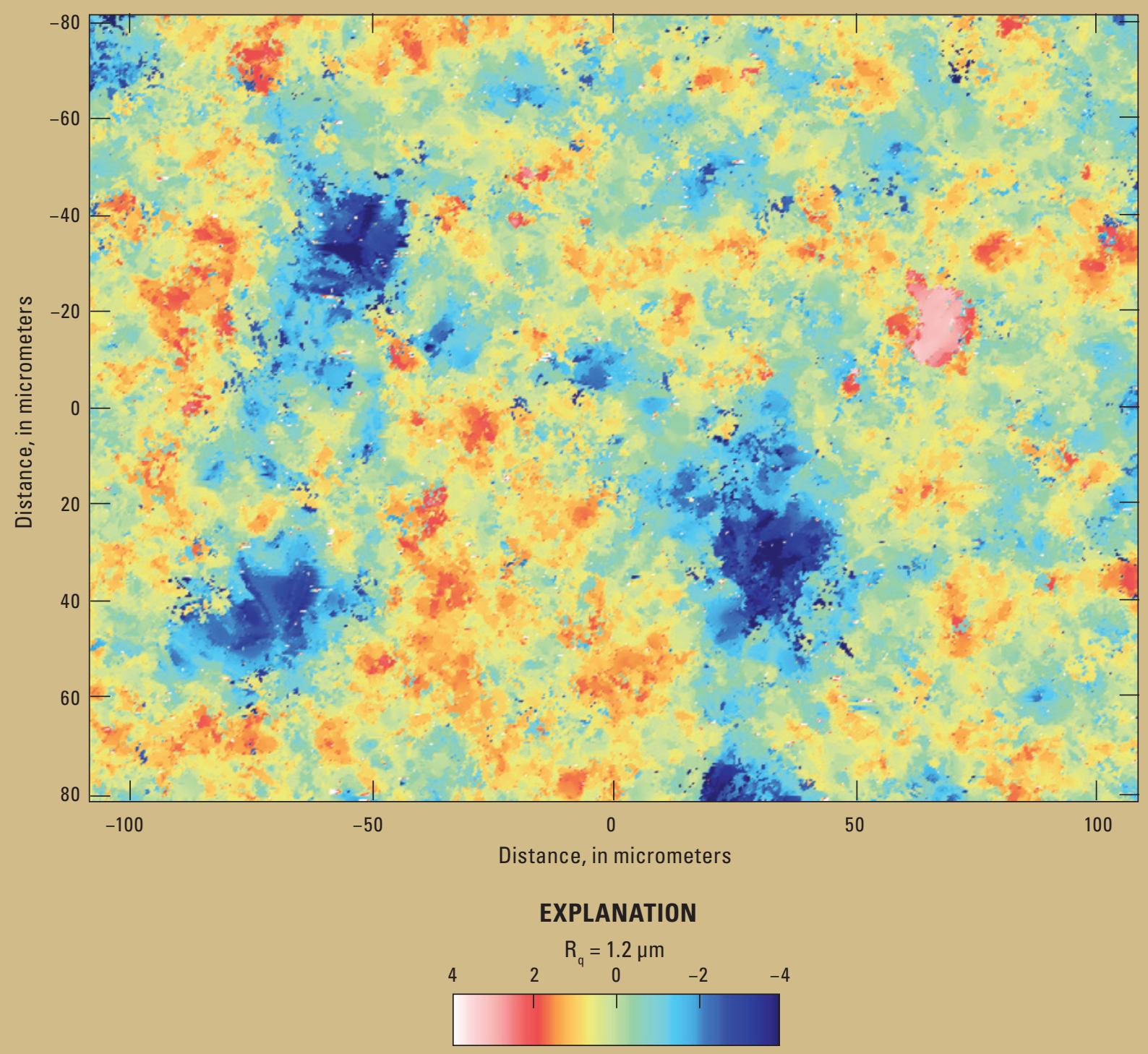

Open-File Report 2020-1134 
Cover. False color image of surface height of ground quart such as what is used in laboratory faulting experiments. $R_{q}$, root mean squared height, $\mu \mathrm{m}$, micrometer. 


\section{Characterizing Fault Roughness-Are Faults Rougher at Long or Short Wavelengths?}

By Nicholas M. Beeler

Open-File Report 2020-1134 


\section{U.S. Geological Survey, Reston, Virginia: 2021}

For more information on the USGS - the Federal source for science about the Earth, its natural and living resources, natural hazards, and the environment-visit https://www.usgs.gov or call 1-888-ASK-USGS.

For an overview of USGS information products, including maps, imagery, and publications, visit https://store.usgs.gov.

Any use of trade, firm, or product names is for descriptive purposes only and does not imply endorsement by the U.S. Government.

Although this information product, for the most part, is in the public domain, it also may contain copyrighted materials as noted in the text. Permission to reproduce copyrighted items must be secured from the copyright owner.

Suggested citation:

Beeler, N.M., 2021, Characterizing fault roughness-Are faults rougher at long or short wavelengths?: U.S.

Geological Survey Open-File Report 2020-1134, 15 p., https://doi.org/10.3133/ofr20201134.

ISSN 2331-1258 (online) 


\section{Contents}

Abstract

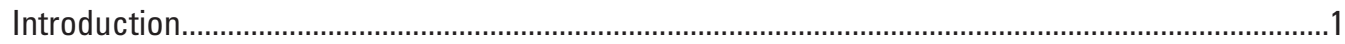

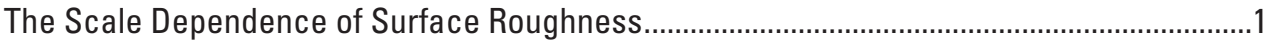

Synthetic Rough Surfaces ..............................................................................................

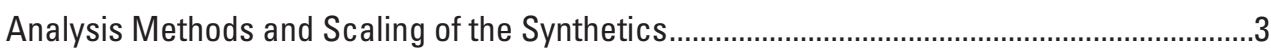

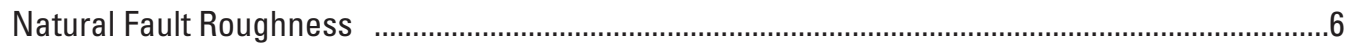

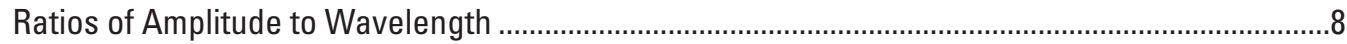

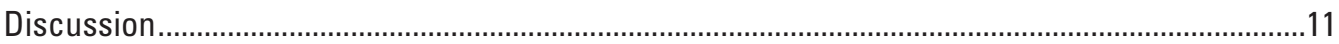

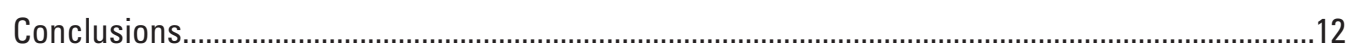

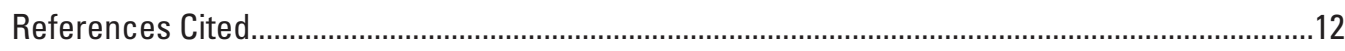

Appendix. Voss-McCartney Algorithm for Pink Noise _....................................................................14

\section{Figures}

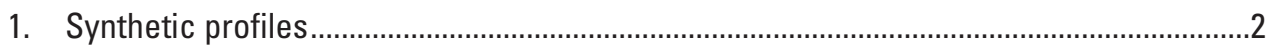

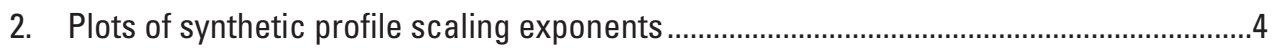

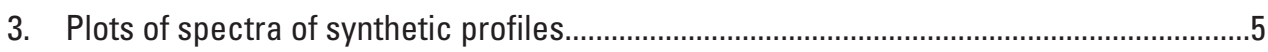

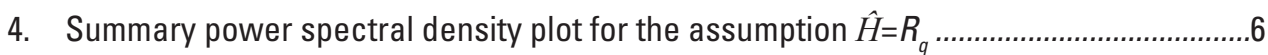

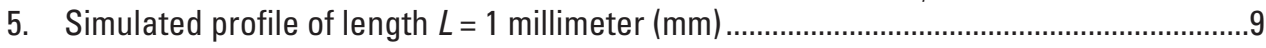

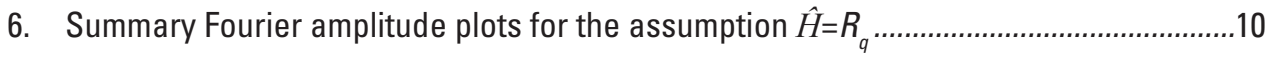

7. Plot of normalized Fourier amplitudes versus wavelength ..............................................11

\section{Tables}

1. Scaling exponents for synthetic surfaces, in descending degree of correlation ............6

2. Summary of scaling exponents in descending degree of correlation for different cases under the standard assumption that average surface height $(\hat{H})$ is the root mean square average roughness, $R$ 


\section{Conversion Factors}

U.S. customary units to International System of Units

\begin{tabular}{|c|c|c|}
\hline Multiply & By & To obtain \\
\hline \multicolumn{3}{|c|}{ Length } \\
\hline inch (in.) & 2.54 & centimeter $(\mathrm{cm})$ \\
\hline inch (in.) & 25.4 & millimeter $(\mathrm{mm})$ \\
\hline foot (ft) & 0.3048 & meter $(\mathrm{m})$ \\
\hline mile (mi) & 1.609 & kilometer (km) \\
\hline mile, nautical (nmi) & 1.852 & kilometer $(\mathrm{km})$ \\
\hline yard (yd) & 0.9144 & meter (m) \\
\hline
\end{tabular}

\section{Abbreviations}

$\begin{array}{ll}\mathrm{MPa} & \text { megapascals } \\ \mu \mathrm{m} & \text { micrometers } \\ \mathrm{mm} & \text { millimeters } \\ \mathrm{RMS} & \text { root mean square }\end{array}$




\title{
Characterizing Fault Roughness-Are Faults Rougher at Long or Short Wavelengths?
}

\author{
By Nicholas M. Beeler
}

\begin{abstract}
Changes in fault roughness with scale, "scaling," is the topic of this report; changes are considered using a general power law relation between some measure of surface height, $H$, and another of length, $L, H=k L^{n}$, where $k$ is a constant and $n$ is an exponent that characterizes the scaling. Extensive profile measurements of natural fault surfaces show that the ratio of average surface height to profile length decreases with scale. Average height is defined using the root mean squared height, $R_{q \text {. }}$ For this analysis, fault surfaces are smoother at long wavelengths (have smaller average height to profile length ratios) than they are at shorter wavelengths. These and other statistical properties of natural fault surfaces hold for more than five orders of magnitude, a huge range from tens of micrometers to 10 meters. However, a different roughness metric, the average height (amplitude) that is specifically associated with a wavelength shows the opposite sense of scaling. The ratio of average amplitude to wavelength increases with wavelength. Thus, the same fault surface can be deemed rougher at long wavelength, or smoother, depending on the chosen metric. This apparent contradiction is a curiosity of the statistics of rough surfaces that have scaling exponents that relate profile length to $R_{\mathrm{q}}$ between 0.5 and 1 , as most natural faults do. To add context, the implied roughness scaling for reference synthetic surfaces is determined. These span the natural range of scaling exponents and have moderate to strong point to point amplitude correlation. The potential payoff of expanded descriptions of natural fault roughness and of reference surfaces are improved constraints on physical mechanisms that generate and modify roughness during shear.
\end{abstract}

\section{Introduction}

Roughness is widely thought to contribute significantly to the shear strength of natural faults (for example, Chester and Chester, 2000). For simplified model faults, such as two bare rock surfaces in contact, the fault shear resistance consists of two components, that due to frictional slip on a planar fault surface, and that which results from the roughness (Chester and Chester, 2000; Dieterich and Smith, 2009; Dunham and others 2011; Fang and Dunham 2013). For more realistic model faults, such as those containing a finite thickness of granular fault gouge bounded by rough surfaces, the roughness likely influences the degree of shear localization within a shear zone, contributing to sliding stability as well as shear strength. Despite these important considerations, little is known about the actual mechanical contributions of roughness to fault slip and earthquake faulting. Much work has focused on characterizing the roughness of exhumed fault surfaces (Power and Tullis, 1991; Candela and others, 2009), and a notable characteristic of fault roughness is that it changes with the spatial scale of observation (for example, Candela and others, 2009). Much less is known about what particular physical mechanisms determine natural fault roughness, and virtually nothing is known about how physical processes lead to the observed changes in roughness with scale of observation (Brodsky and others, 2011). The purpose of this report is to improve methods of characterizing fault roughness and to better define how roughness changes with spatial scale.

\section{The Scale Dependence of Surface Roughness}

Throughout this report roughness refers to a ratio of surface height, $H$, to length, $L$. As you will see using this definition, roughness $(H / L)$ is a property that depends critically on the definitions of height and length used. Changes in fault roughness with scale, "scaling," is the topic of this report; changes are considered using a general power law relation between surface height and length,

$$
H=k L^{n}
$$

where $k$ is a constant and $n$ is an exponent that characterizes the scaling roughness metrics (for example, see Brodsky and others, 2011).

Early modern work on scaling suggested that surfaces were self-similar (Power and Tullis, 1991). Self-similarity, described in more detail in the Natural Fault Roughness section of this report, is a theoretical construct of Mandelbrot (1967), which for surface roughness requires the average height to profile length ratio remain constant-in other words, roughness is scale independent. This definition can be accommodated in the general form of roughness scaling (eq. 1) by replacing $H$ with the average, $\hat{H}$, and $L$ with the profile length, $L_{p}$, across which the average is taken (Brodsky and others, 2011), 


$$
\widehat{H}=k_{l p} L_{P}^{\zeta}
$$

with scaling exponent, $\zeta$. For these choices of representative average height, profile length, and if the average is equated with the root mean square (RMS; see below), then $\zeta$ is known as the Hurst exponent (Mandelbrot and van Ness, 1968). Selfsimilar surfaces have $\zeta=1$. Equation 2 with RMS average height is the most common and standard metric to define the scaling of roughness (Power and Tullis, 1991; Candela and others, 2012).

For contrast and comparison, we use a different roughness metric, the average height or amplitude, $\hat{a}$, of points on the fault surface averaged over a particular wavelength, $\lambda$. If fault roughness is not exactly self-similar, it changes with scale. So, in addition to profile length-dependent heights there is value in specific surface amplitudes, and specific wavelengths as well as, if not more so than, profile lengths. To contrast with the scaling defined in equation 2 , here is an alternative form of the general power law (eq. 1),

$$
\hat{a}=k_{\lambda} \lambda^{m}
$$

with scaling exponent $m$. Note that comparing the roughness scaling of profiles using equation 2 to that of equation 3 implies no judgment about which is "correct;" they both are correct but use different descriptions of the profile or surface. Throughout this report, equations 2 and 3 are referred to as different scaling metrics.

\section{Synthetic Rough Surfaces}

To illustrate roughness scaling and some of the statistical and signal analyses that can be used to determine the scaling exponents, reference synthetic profiles are constructed and analyzed. The three different types of synthetics are designed to have known values of the scaling exponents $\zeta(0$ to 1$)$ in equation 2 and $m(1 / 2$ to $3 / 2)$ in equation 3 . Unlike prior studies, where synthetic fault profiles were generated using one of the same techniques used to analyze them (for example, the Fourier transform; Candela and others, 2009), here the profiles are generated from random numbers.

A highly correlated but randomly generated synthetic profile can be constructed by assuming a random walk (fig. $1 A$ ): the synthetic trace is produced by sequentially assigning the amplitude of each point, $a(i)$, to be the amplitude of the prior point $a(i-1)$ plus a random amplitude. The random component is the product of a chosen scaling value, $a_{c}$ and a value selected from a uniform distribution between -1 and 1 using a random generator. Thus, the walk increments are uniformly distributed between $-a_{c}$ and $+a_{c}$. This is a well-known procedure to produce Brown noise (for example, Voss and Clarke, 1978). The example reference profile has 6,400 points, $a_{c}=0.3$ micrometers $(\mu \mathrm{m}), L_{p}=215 \mu \mathrm{m}$, and a linear trend has been removed so that the mean profile normal distance is 0 .

A less but still notably correlated synthetic profile can be constructed from the running sum of a fixed number of random sequences, where each sequence has a different
A. Moderately correlated sythentic random surface (Brown noise)



B. Poorly correlated synthetic random profile (pink noise)

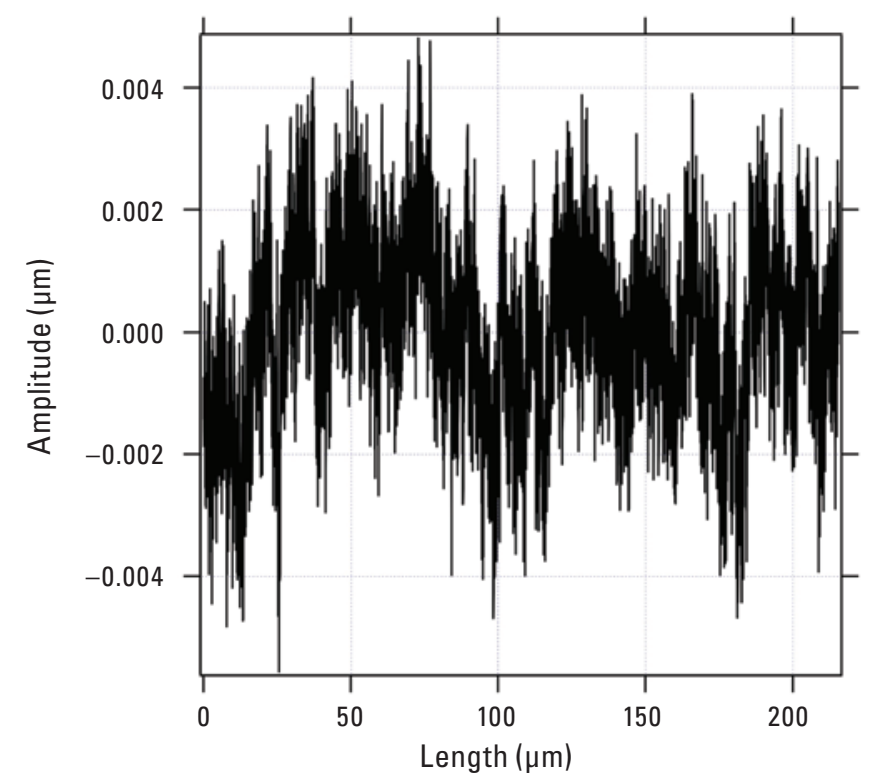

Figure 1 ( pages 2-3). Plots of simulated synthetic profiles. A, Brownian noise. The profile was generated using a random walk along a profile length of 215.5 micrometers $(\mu \mathrm{m})$, with a walk step taken every $0.34 \mu \mathrm{m}$. There are 6,400 points in the profile. The steps are chosen randomly from a uniform distribution between -0.3 and $0.3 \mu \mathrm{m}$. Once the profile generation is complete, any overall linear trend is removed. $B$, Pink noise. The profile was generated using the Voss-McCartney algorithm (see Gardner, 1978), as described in the appendix. $C$, Self-similar profile generated from integrating the pink noise in part $B$ and rescaling the amplitudes. 


\section{Highly correlated synthetic random profile (self-similar)}

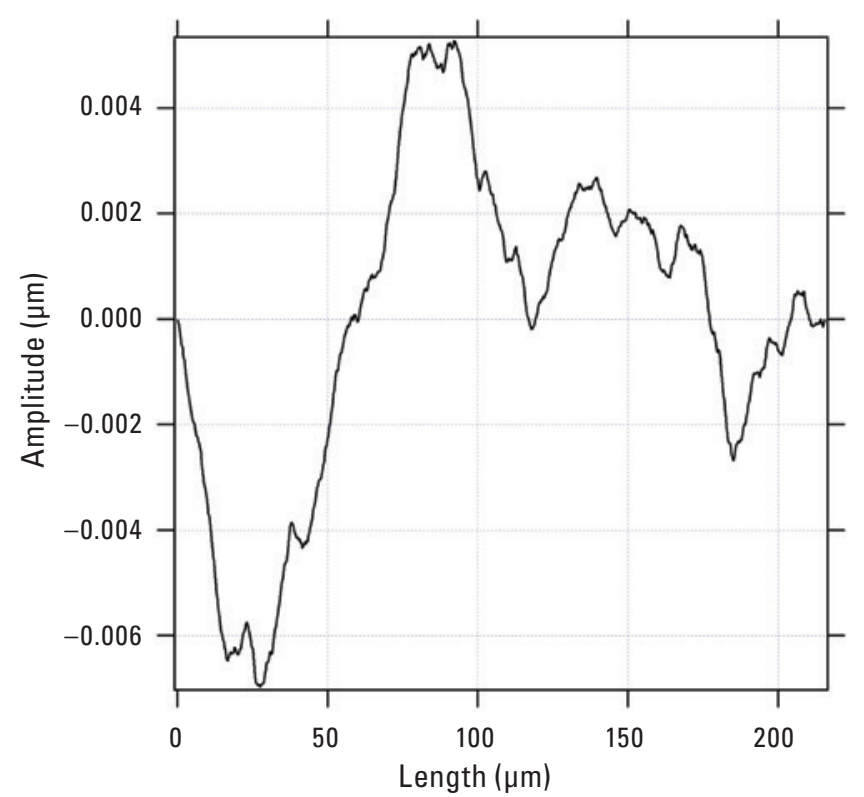

Figure 1 ( pages 2-3).-Continued

update frequency. The algorithm, which is in part described by Gardner (1978), originates from Voss and Clarke (1978) and is intended to produce pink noise. This implementation, detailed in the appendix, is a modification of Voss's original based on one by James McCartney. ${ }^{1}$ The strategy is to reduce the Brownian correlation but not to the degree that adjacent points in the profile are entirely independent (white noise). Instead of following the Brownian sequence, where a random increment is added to the cumulative sum at each step, the sum for the Voss-McCartney algorithm is continuously and incrementally altered at a comprehensive range of update periods (fig. $1 B$ ). In the figure $1 B$ example, each random number is selected from a Gaussian distribution that has a standard deviation of $0.4 \mu \mathrm{m}$. This profile has $16,383\left(=2^{12}-1\right)$ points, $L_{p}=215 \mathrm{~mm}$, and the linear trend has been removed so that the mean profile normal distance is 0 .

A more strongly correlated profile can be generated by numerically integrating the amplitude of an existing synthetic profile across length and rescaling or redimensioning the resulting amplitudes. Doing so increases the scaling exponent by 1 . The exponent determines the degree of correlation between adjacent points along the profile length, and correlation increases with the exponent. For example, for equation 3 with $m=0$, amplitudes would be completely uncorrelated. The third reference is generated by integrating the pink noise profile, resulting in a profile (fig. $1 C$ ) that is more correlated than pink or brown noise. The result has $16,383\left(=2^{12}-1\right)$

\footnotetext{
${ }^{1}$ Refers to an unpublished correspondence from J. McCartney addressed to the Music and Digital Signal Processing mailing list from September 1999.
}

points, $L_{p}=215 \mathrm{~mm}$, and the amplitude has been rescaled/ redimensioned by normalizing by $L_{p} / 5$. The rescale choice is so that the amplitude range is like the other references but is otherwise arbitrary.

\section{Analysis Methods and Scaling of the Synthetics}

Throughout this section and in subsequent parts of this report, different analysis techniques are used to characterize scaling. These are methods to evaluate the metrics (eqs. 2 and 3 ) that define the aspects of roughness scaling. Of interest are the scaling exponents, $\zeta$ and $m$, in these equations. To infer the scaling exponent for the profile-length-averaged height metric (eq. 2), requires first removing the mean height at each profile length (for example, Schmittbuhl and others, 1995, and references therein). Qualitatively, the necessary procedure is to define some measure of the average deviation of the surface across the profile length, such as in formal statistical definitions of variance. Using the uncorrected standard deviation

$$
d_{s u}=\sqrt{\frac{1}{N} \sum_{i=1}^{N}\left(a_{i}-\bar{a}\right)^{2}}
$$

at each possible profile length (wavelength) between 2 and N/2 points and averaging all values at each length produces a good estimate of the expected exponent for the Brown noise profile (fig. 2A) (for example, Schmittbuhl and others, 1995). How the average amplitude changes with profile length is controlled by the Hurst exponent, $\zeta$. Here, $a_{i}$ is the amplitude at each point, $N$ is the total number of points in the profile length of interest, and $\bar{a}$ is the average amplitude across the profile length,

$\bar{a}=\frac{1}{N} \sum_{i=1}^{N} a_{i}$.

Similarly, the corrected standard deviation,

$$
d_{s}=\sqrt{\frac{1}{N-1} \sum_{i=1}^{N}\left(a_{i}-\bar{a}\right)^{2}}
$$

and the average deviation about the mean,

$$
d_{a}=\frac{1}{N} \sum_{i=1}^{N}\left|a_{i}-\bar{a}\right|
$$

retrieve viable estimates of the scaling exponent (fig. $2 A$ ), as do several other approaches (for example, Schmittbuhl and others, 1995; Candela and others, 2009). For all deviation methods (eqs. 4, 5, and 6), the slopes agree and are consistent with the expected exponent for the Brown surface at profile lengths between 0.1 and $10 \mu \mathrm{m}$. At longer wavelengths, the exponent is not resolved. At shorter wavelengths, the standard deviation fails to resolve the exponent.

For the Brown profile, the Hurst exponent is essentially $1 / 2$, as expected for a random walk (Mandelbrot and van Ness, 1968). The pink profile has a weak and poorly defined scaling exponent using these analysis methods (fig. $2 B$, see discussion in Candela and others, 2009). In marked contrast, the third reference has a Hurst exponent of approximately 1 (fig. 2C) and 


\section{A. Brown noise profile}

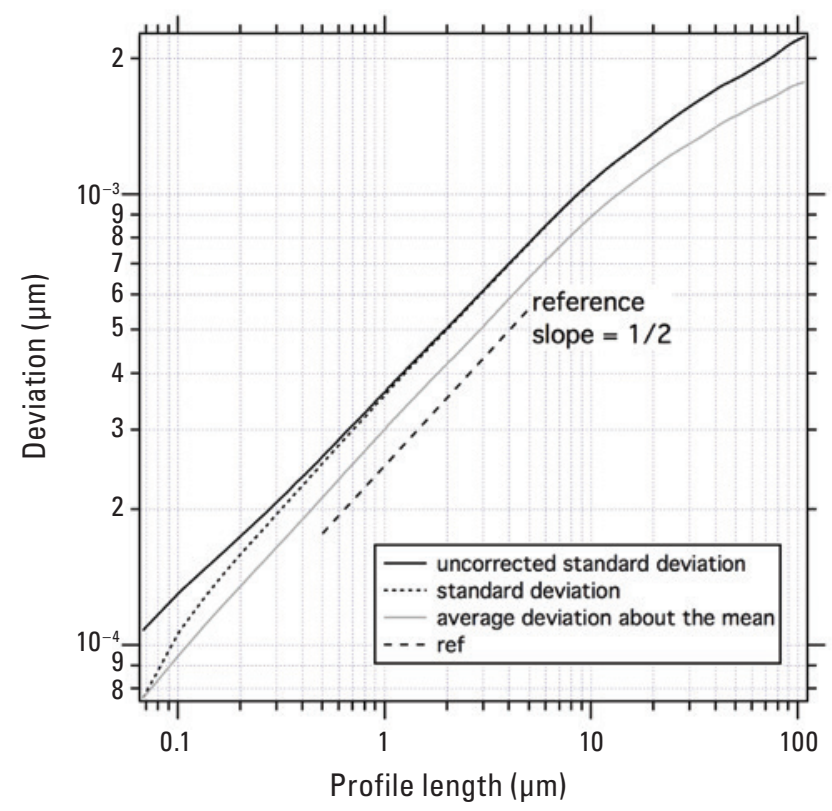

C. Self-similar profile

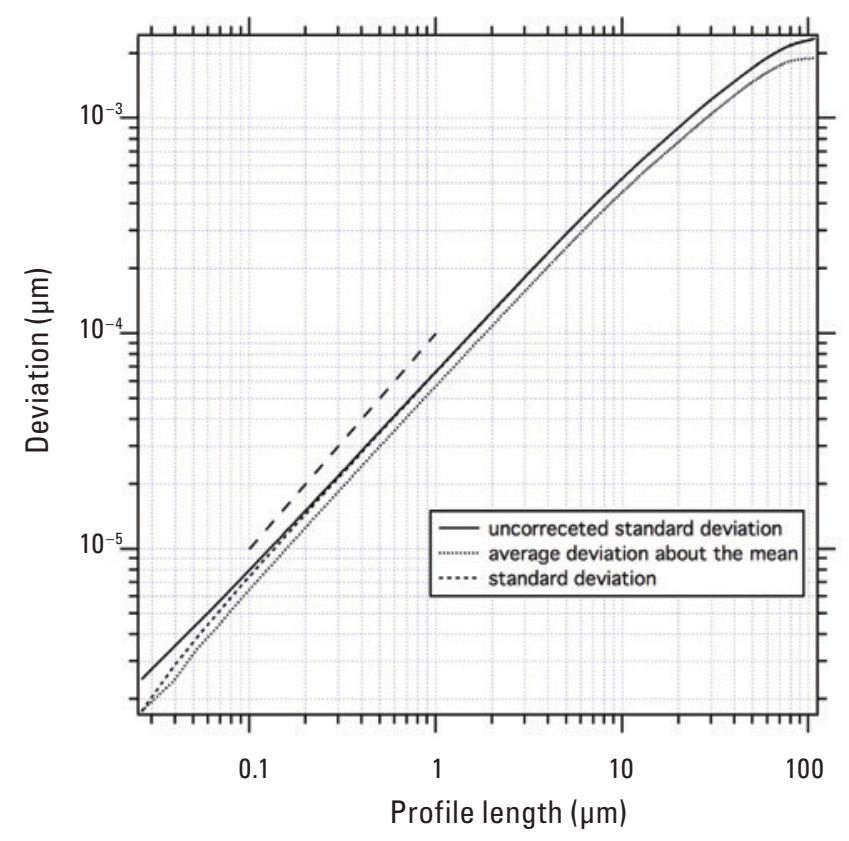

is self-similar (Mandelbrot, 1967). Allowing that a pink profile is uncorrelated $(\zeta=0)$ for this metric (eq. 2), the self-similar profile would be consistent with its genesis, namely that the integral of the profile with no scaling requires a scaling exponent of 1 . Though this remains to be seen, the integrated result is a highly correlated profile. The Hurst exponents of the three synthetic profiles are summarized in table 1 where subsequent analysis (see below) is used to infer the scaling of pink noise.

Rather than using an average deviation across the profile length to determine the scaling exponent of equation 2 , the scaling exponent for the metric that relates specific amplitudes
B. Pink noise profile

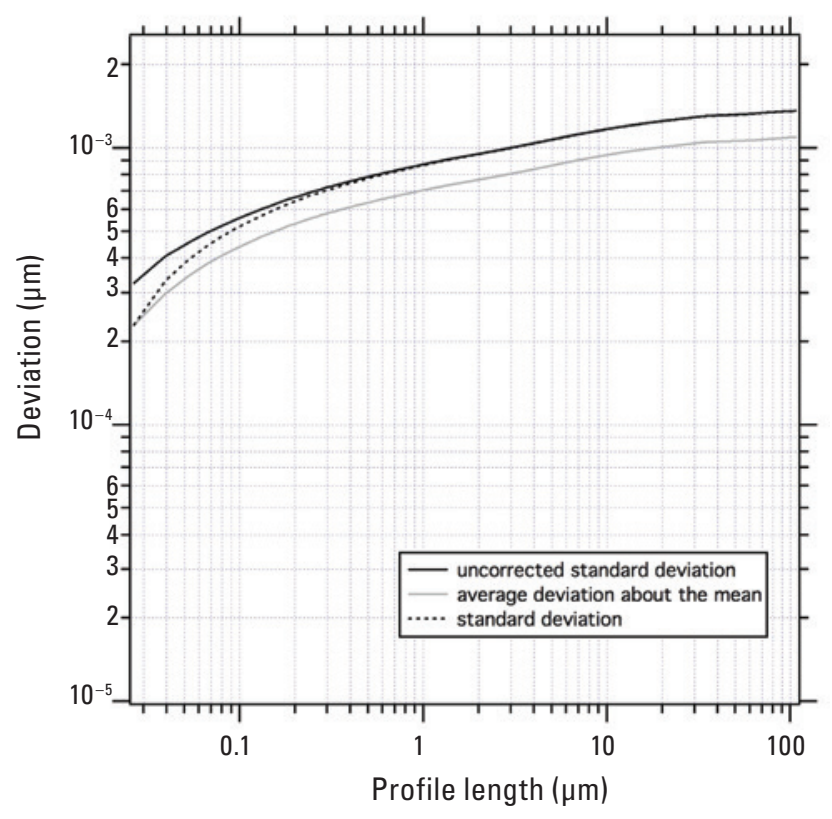

Figure 2. Plots of synthetic profile scaling exponents. Plots are of the deviation from the mean profile amplitude as a function of the profile length. The slope of the plot is an estimate of the scaling exponent relating profile length to average height (see eq. 2). Three estimates of the deviation are shown: the standard deviation (dotted line), the uncorrected standard deviation (solid black line), and the average deviation about the mean (solid gray line). $A$, Brownian noise with reference slope of $1 / 2$ (dashed line). $B$, Pink noise. $C$, Self-similar with reference slope of 1 (dashed line). $\mu \mathrm{m}$, micrometers.

and wavelengths, equation 3, is determined using the Fourier transform (fig. 3). Throughout this report, the transforms shown are untapered (raw) and therefore have not accounted for the effects of spectral leakage. Leakage can lead to substantial bias in estimates of the scaling exponent (for example, Barbour and Parker, 2014). This report is limited to a qualitative discussion of scaling, rather than precise determinations of the exponent.

For well-resolved spectra, the scaling exponent in the physical realm, $m$, is preserved in the spatial frequency domain (see section on Ratios of Amplitude to Wavelength, below, for a detailed example): 


\section{A. Brown noise}

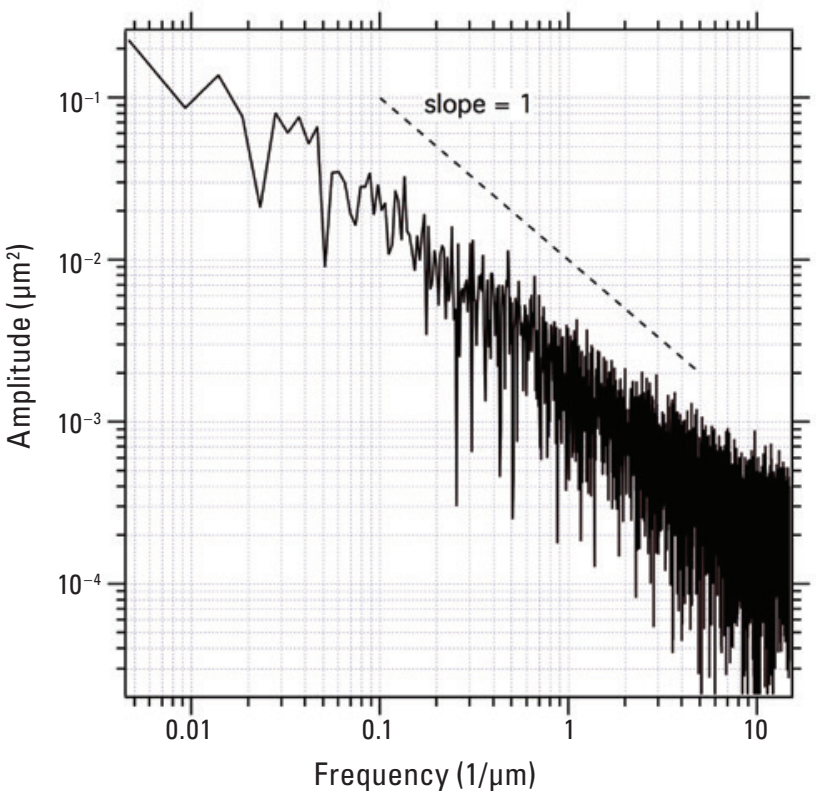

C. Self-similar

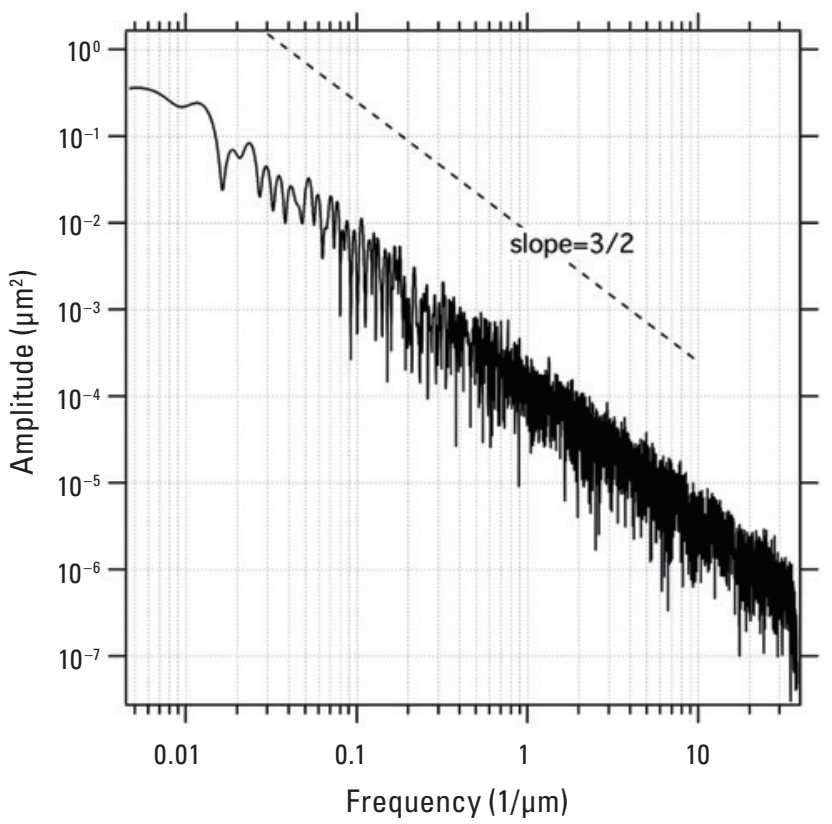

$A \propto \lambda^{m}$

where $A$ is the amplitude of the transform, referred to throughout as Fourier amplitude to distinguish it from the physical average, wavelength-specific amplitude . Noting the inverse relation between wavelength and spatial frequency, $f_{s}=1 / \lambda$, Brown noise has a Fourier amplitude spectrum that depends on frequency as $1 / f_{s}$ (proportional to $\lambda$ ). Therefore, in equation 7, $m=1$ and $A / \lambda \propto 1$ for the moderately correlated profile. Equivalently, the ratio of amplitude to wavelength, $\hat{a} / \lambda$, is independent of wavelength (see fig. $3 A$ ). Pink noise has a Fourier amplitude spectrum that depends on frequency as

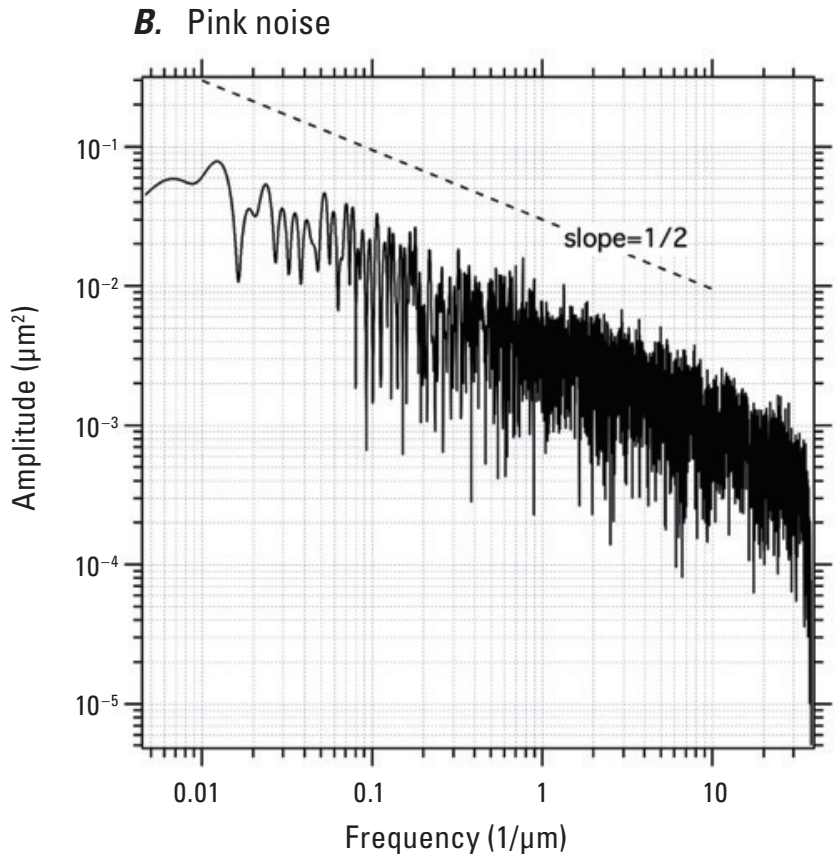

Figure 3. Plots of spectra of synthetic profiles. Plots are of the Fourier amplitude (in square micrometers $\left[\mu \mathrm{m}^{2}\right]$ ) as a function of spatial frequency (in inverse micrometers [ $1 / \mu \mathrm{m}]$ ). The slope of the plot, shown by the dashed line, is an estimate of the scaling exponent relating wavelength to average height (see eq. 3). $A$, Brown noise with reference slope of 1 . B, Pink noise with reference slope of $1 / 2$. $C$, Self-similar with reference slope of $3 / 2$.

$1 / \sqrt{f_{s}}$ (as $\sqrt{\lambda}$ ) (see fig. $3 B$ ). In this case in equation 7 , $m=1 / 2$ and $A / \lambda \propto 1 / \sqrt{\lambda}$ for the moderately correlated profile. Equivalently, the ratio of amplitude to wavelength, $\hat{a} / \lambda \propto 1 / \sqrt{\lambda}$, depends on wavelength (fig. $3 B$ ). For this roughness metric, pink profiles have roughness that decreases with wavelength. The self-similar profile has Fourier amplitudes that depend on frequency as $f^{-\frac{3}{2}}\left(\right.$ as $\left.\lambda^{3 / 2}\right)$ such that $A / \lambda \propto \sqrt{\lambda}$, equivalently $\hat{a} / \lambda \propto \sqrt{\lambda}$. By this metric, roughness of a selfsimilar profile increases with wavelength, rather than being independent of scale, as required by the standard metric in equation 2 . The scaling exponents $m$ of the three synthetic profiles are summarized in table 1. 
Table 1. Scaling exponents for synthetic surfaces, in descending degree of correlation (descending exponent).

[ $\zeta$ is the exponent in the scaling relation between average surface height and profile length, $\hat{H} \propto L_{p}^{\zeta}$ (see eq. 2). $m$ is the exponent in the scaling relation between average amplitude and specific wavelength, $\hat{a} \propto \lambda^{m}$ (see eq. 3)]

\begin{tabular}{lcc}
\hline \multicolumn{1}{c}{ Case } & $\boldsymbol{\zeta}=\boldsymbol{m} \mathbf{- 1 / 2}$ & $\boldsymbol{m}=\boldsymbol{\zeta + 1 / 2}$ \\
\hline Self-similar & 1 & $3 / 2$ \\
Brown noise & $1 / 2$ & 1 \\
Pink noise & 0 & $1 / 2$ \\
\hline
\end{tabular}

\section{Natural Fault Roughness}

Whereas Power and Tullis (1991) inferred that natural faults were self-similar, subsequent work using higher resolution and a broader range of wavelengths shows that self-similarity does not strictly hold and instead of being constant, $\hat{H} / L_{p}$ changes with scale (Candela and others, 2012). The most widely used analysis technique to determine scaling of fault surface profiles, which relates to standard roughness metrics (for example, $R_{q}$, defined below), self-similarity, and the Hurst exponent, is to calculate the power spectral density $\left(P_{s d}\right)$ of the profile (fig. 4). Power spectral density is the square of the magnitude of the Fourier transform of the profile normalized by the profile length, $L_{p}$. The surface $P_{s d}($ fig. 4 ) also follows a power law

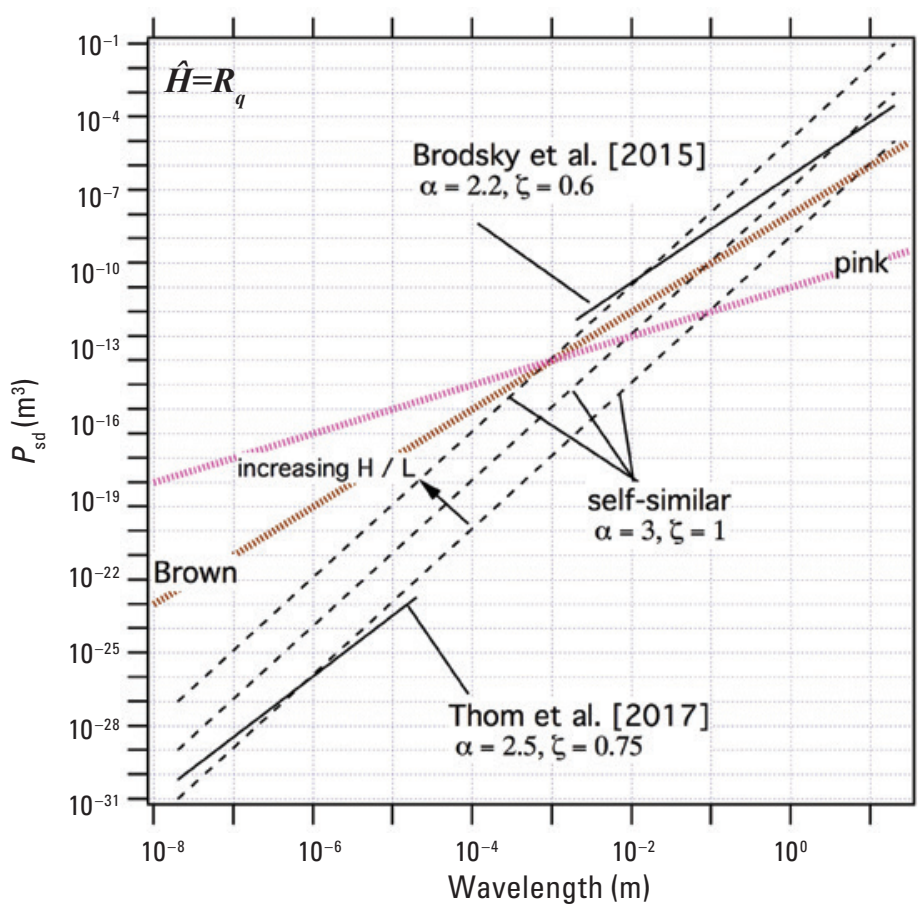

$$
P_{s d}=c \lambda^{\alpha}
$$

where $c$ and $\alpha$ are empirically determined. Note that since $m$ is the amplitude to wavelength slope measured in the Fourier transform of the profile (fig. 3), and the power spectral density is the normalized square of the transform, then the exponents $m$ (eqs. 3 and 7) and $\alpha$ (eq. 8) relate as $m=\alpha / 2$.

The relations among the generic $P_{\text {sd }}$ (eq. 8), the standard scaling relation (eq. 2 ), its scaling exponent $\zeta$, and the justification for using RMS as the average height come from an application of Parseval's theorem (Power and Tullis, 1991; Chatfield, 1975). The theorem states that the integral of a function squared is equal to the integral of its transform squared. In this case, taking a profile of surface amplitude $g(\lambda)$ and its transform $G\left(f_{\mathrm{s}}\right)$, where $f_{\mathrm{s}}$ is the spatial frequency $f_{s}=1 / \lambda$, the theorem requires

$$
\int_{0}^{\left(L_{p}\right)} g(\lambda)^{2} d \lambda=\int_{1 / L_{p}}^{\infty} G\left(f_{s}\right)^{2} d f_{s}
$$

Here, as is inevitable in signal processing of finite series, the infinite limits in the formal definition of the transform ( $-\infty$ to $+\infty)$ are replaced with the finite bounds of the profile ( 0 to $\left.L_{p}\right)$. This is equivalent to assuming that the spatial series entirely defines the frequency content of interest-for example, if the finite signal repeats infinitely in the positive and negative directions beyond the ends of the profile, or, alternatively, that

Figure 4. Summary power spectral density plot for the assumption $\hat{H}=R_{q}$. Two natural fault surfaces area shown schematically in black (Brodsky and others, 2015; Thom and others, 2017). Example spectra of self-similar surfaces are shown as dashed lines of slope $\alpha=3$, corresponding to constant average-height-to-profile-length ratios. Lines for pink $(\alpha=1)$ and Brown noise $(\alpha=2)$ are shown for reference. $\hat{H}$, average surface height; $R_{\mathrm{q}^{\prime}}$ root mean square average roughness ; m, meters. 
the series is truly infinite but that there is zero amplitude at distances less than zero and longer than $L_{p}$.

The square root of the left-hand side of equation 9 normalized by $L_{p}$ is the definition of the RMS roughness, $R_{\mathrm{q}}$, a standard engineering surface measure. On the right-hand side, $G\left(f_{\mathrm{s}}\right)^{2}$ normalized by profile length is $P_{\text {sd }}$. Thus, making these substitutions results in

$$
R_{q}=\sqrt{\int_{1 / L_{p}}^{\infty} P_{s d} d f_{s}}
$$

As noted in the Introduction section, the standard approach (for example, Brodsky and others, 2011, 2015) is to equate $R_{\mathrm{q}}$ (eq. 10) with the average height $\hat{H}$, and substituting equation 8 into equation 10 results in

$$
\widehat{H}=\sqrt{\int_{1 / L_{p}}^{\infty} c f_{s}^{-\alpha} d f_{s}}
$$

Carrying out the integration and evaluating yields (Brodsky and others, 2011, 2015):

$$
\widehat{H}=\sqrt{\frac{c}{\alpha-1}} L_{p}^{(\alpha-1) / 2}
$$

To complete the relations, equating the exponents in equations 2 and $12, \alpha=1+2 \zeta$, shows that $P_{\text {sd }}$ can be recast to infer the Hurst exponent from a plot of the power spectra density versus wavelength (Power and Tullis, 1991; Brodsky and others, 2011, 2015),

$$
P_{s d}=c \lambda^{(1+2 \zeta)}
$$

Here the exponent has been substituted back into equation 2 .

Recalling that the requirement for scale independence (self-similarity) is that the scaling exponent is 1 , on the power spectral plot, self-similar surfaces have a slope of 3 (Power and Tullis, 1991). Increasing ratios (increasing values of $\hat{H} / L_{p}$ ) correspond to increasing $P_{\text {sd }}$, as shown in figure 4 . In contrast, high resolution studies of natural fault surfaces have power spectral slopes that are less than 3 (Brodsky and others, 2011; Candela and others, 2012), requiring scaling exponents less than 1 . Schematic representations of recently published results in figure 4 are $\zeta=0.6$ (Candela and others, 2012; Brodsky and others, 2015) and $\zeta=0.75$ (Thom and others, 2017), corresponding to $\alpha=2.2$ and $\alpha=2.5$, respectively. If these surfaces are considered in the context of fixed $\hat{H} / L_{p}$ ratios, such as the three dashed example lines in figure 4, higher wavelengths correspond to smaller height-to-length ratios. By this metric, the surfaces are smoother at long wavelengths than they are at shorter wavelengths.

Models of the evolution or genesis of natural fault roughness and its scaling are contingent on assumptions about rock fracture, initial roughness, and surface wear, among other things (Renard and Candela, 2017). However, there are only a few observations of the roughness of laboratory shear failures in rock or observations of the slip dependence of roughness in the laboratory or field. Chen and Spetzler (1993) found that shear fractures of Westerly Granite generated under modest confinement of 100 megapascals (MPa) are Brownian with $\alpha=2(\zeta=1 / 2)$; subsequent slip on these fractures was between 100 and $800 \mu \mathrm{m}$, a small amount on the order of the grain size, and did not alter the scaling. Amitrano and Schmittbuhl (2002) produced shear fractures in Sidobre granite at confining pressures between 20 and $80 \mathrm{MPa}$, resulting in higher scaling exponents in the shearing direction than in the previous study, such that $\alpha=2.5(\zeta=0.74)$. They also report a slight increase in the scaling exponent with slip. Noting that many natural faults originate from tensile fractures, notably joints, Davidesko and others (2014) sheared mated 15-centimeterlong tensile fractures of fine-grained limestone as much as 15 millimeters ( $\mathrm{mm}$ ) of slip (unconfined) and found scaling exponents approaching self-similarity, such that $\alpha=2.7-2.95$ $(\zeta=0.85-0.98)$. The roughness decreased at all wavelengths progressively with slip, but the scaling exponent did not change appreciably. Smoothing of fault surfaces, with no change in the scaling exponent, is also observed in the field (Brodsky and others, 2011), and most comprehensively by Dascher-Cousineau and others (2018) for faults in sandstone with $\zeta=0.4$ in the direction of shear. Noting that the largest dimension faults have the largest displacements, a strong argument that scaling is independent of shear displacement are observations that scaling is independent of fault length (for example, Renard and Candela, 2017).

This report's interest in characterizing the scaling notwithstanding, it is the absolute amplitude of roughness that affects the shear resistance in simulations (for example, Dunham and others, 2011, and references therein), at least for initially mated surfaces at short displacement. Accordingly, on Earth the absolute fault surface amplitude and how it changes with slip is thought to determine the structure (shear zone and adjacent damage zone thicknesses) and to influence the strength and other physical properties of natural shear zones. Comparisons between natural faults (fig. 4) and the synthetic profiles (figs. 1, 2, and 3) using equation 2 are useful since the synthetic profiles span the fairly wide and somewhat inconsistent range of natural and experimental scaling exponents $(\zeta=0.4-0.98)$, and because much is known about the genesis of the synthetic profiles and less for the natural cases. In addition to the self-similar curves that correspond to the scaling of the integrated pink noise synthetic profile (dashed line in fig. 4), superimposed on the power spectra schematic plot in figure 4 are reference lines that represent the scaling for pink $(\alpha=2 m=1)$ (pink-striped line) and Brown noise $(\alpha=2 m=2)$ (brown-striped line).

Taking the point of view that the random walk doesn't correspond to any known physical mechanism of roughness generation, it is reassuring that natural fault examples generally do not show the same scaling. Most natural faults are more correlated than a random walk, suggesting even more structure to their genesis, keeping in mind that a random walk is a highly correlated random profile. The natural examples in figure 4, which are typical for faults in crystalline rock, are not dramatically different than Brownian surfaces, particularly 
those of Brodsky and others (2015) and Candela and others (2012). Considering their scaling exponents, natural fault surfaces with $\zeta=0.6$ to $0.75(\alpha=2.2$ to 2.5$)$ (Candela and others, 2012; Brodsky and others, 2015; Thom and others, 2017) are arguably more similar to a Brownian surface $(\zeta=0.5, \alpha=2)$ than they are to a self-similar surface $(\zeta=1$, $\alpha=3$ ). Since self-similarity (scale independence) is not consistent with natural fault surfaces, is a mathematical construct, and has no associated mechanical significance, a comparison with self-similarity may provide less constraints on process than the random walk example. Perhaps more revealing in this context is reconsideration of the pink profile and the apparent inconsistency between its construction and the standard analysis procedure. Whereas equation 2 requires $\zeta=0$ for a pink surface, the synthetic profile is constructed to have a specific scale dependence and, even visible to the eye, it has an obvious correlated roughness. A similar problem arises when considering synthetic white noise (not shown), which, according to its construction, has truly uncorrelated amplitudes $(m=0)$. Using the standard roughness metric (eq. 2), the Hurst exponent of a white noise profile is $-1 / 2$, meaning its profile-length-averaged height is anticorrelated.

\section{Ratios of Amplitude to Wavelength}

To restate a point made in the Introduction section, seemingly inconsistent Hurst exponents of synthetic surfaces relative to their method construction is not a question of validity; that pink noise has a Hurst exponent of 0 is a fact. Rather, the issue is whether Hurst exponents are the only valuable representation of roughness scaling. In considering physical mechanisms that control fault roughness and faulting itself, there may be use in metrics that are not cast as profile length averages or depend intrinsically on profile length. For instance, the necessary condition for frictional failure at a point on a fault depends on the stress state, fault strength, and local surface orientation. Local surface orientation about asperities is more directly related to the local slope via the amplitude to wavelength ratio than it is to profile-lengthaveraged height. Accordingly, consider the scaling associated with the physical amplitude at specific wavelengths (eq. 3). The following section contrasts scaling with equation 3 to the standard approach (eq. 2).

Imagine an idealized one-dimensional surface profile in the $\mathrm{x}$-direction, that is made up of the sum of cosine functions at all wavelengths (Power and Tullis, 1991). At any value of $\lambda$, the contribution to the profile is

$$
g(\lambda)=a(\lambda) \cos \left(\frac{2 \pi x}{\lambda}+\phi\right)
$$

where $a$ is the amplitude, here allowed to depend on wavelength, and $\phi$ is a random phase shift. Estimates of the corresponding Fourier amplitude at each wavelength and scaling with wavelength can be made analytically. The Fourier transform of a profile of length $L_{p}$ at a specific frequency (wavelength) is

$$
G(\omega)=\int_{0}^{L_{p}} g(x) e^{-i \omega x} d x
$$

where $\omega$ is the angular spatial frequency, $\omega=2 \pi / \lambda$. In the general case, the transform results in a complex series in the frequency domain, $G(\omega)$. However, for this example construction, the imaginary part can be avoided. Using Euler's formula, equation 15 can be decomposed into the real and imaginary parts,

$$
G(\omega)=\int_{0}^{L_{p}} g(x) \cos (\omega x) d x-\int_{0}^{L_{p}} g(x) i \sin (\omega x) d x
$$

Here, on the right-hand side of equation 16 from left to right, the integrals are the real ( $R$, for short below) and imaginary parts ( $I$, for short) of the transform, respectively. The amplitude, $A$, of the transform is the quantity of interest,

$$
A=\sqrt{R^{2}+I^{2}}
$$

Equation 17 is the formal definition of the Fourier amplitude referred to previously in equation 7 . Fourier amplitude is independent of the phase shift, $\phi$. Therefore, for the choice of cosines and for simplicity, taking $\phi=0$ makes equation 14 an even function. Then there is no imaginary part in the transform and $A=R$. Substituting equation 14 into 16 and then into 17 yields

$$
A=a(\lambda) \int_{0}^{L_{p}} \cos ^{2}\left(\frac{2 \pi x}{\lambda}\right) d x
$$

with the Fourier amplitude

$$
A=a(\lambda)\left(\frac{L_{p}}{2}+\frac{\lambda}{8 \pi} \sin \left(\frac{4 \pi L_{p}}{\lambda}\right)\right)
$$

For the length scales that are not aliased, such as $\lambda / 4 \pi<<L_{p}$, equation 19 is

$$
A \approx \frac{a(\lambda) L_{p}}{2}
$$

Thus, if the amplitudes $a(\lambda)$ of the parts of the profile depend on wavelength, as in equation $3, k_{\lambda} \lambda^{m}$, then from equation 20 , the Fourier amplitudes are

$$
A \approx \frac{k_{\lambda} \lambda^{m} L_{p}}{2}
$$

Figure 5 shows these relations between the physical amplitudes $a$ and Fourier amplitudes $A$ for an example where the total profile length $L_{p}$ is $1 \mathrm{~mm}$. The synthetic profile (fig. $5 A$ and $B$ ) is made up of 21 specific wavelengths that are approximately equally spaced logarithmically between 1 and $1,000 \mu \mathrm{m}$. The amplitudes are shown in figure $5 C$ and obey the power law relation with $k_{\lambda}=0.01, m=1$, and $a=0.01 \lambda$. The individual wavelengths also have random phase shifts. The analytical estimates of Fourier amplitude (eqs. 19 and 20) from the prescribed physical amplitudes and wavelengths are shown in figure $5 D$, as are those estimated numerically from 

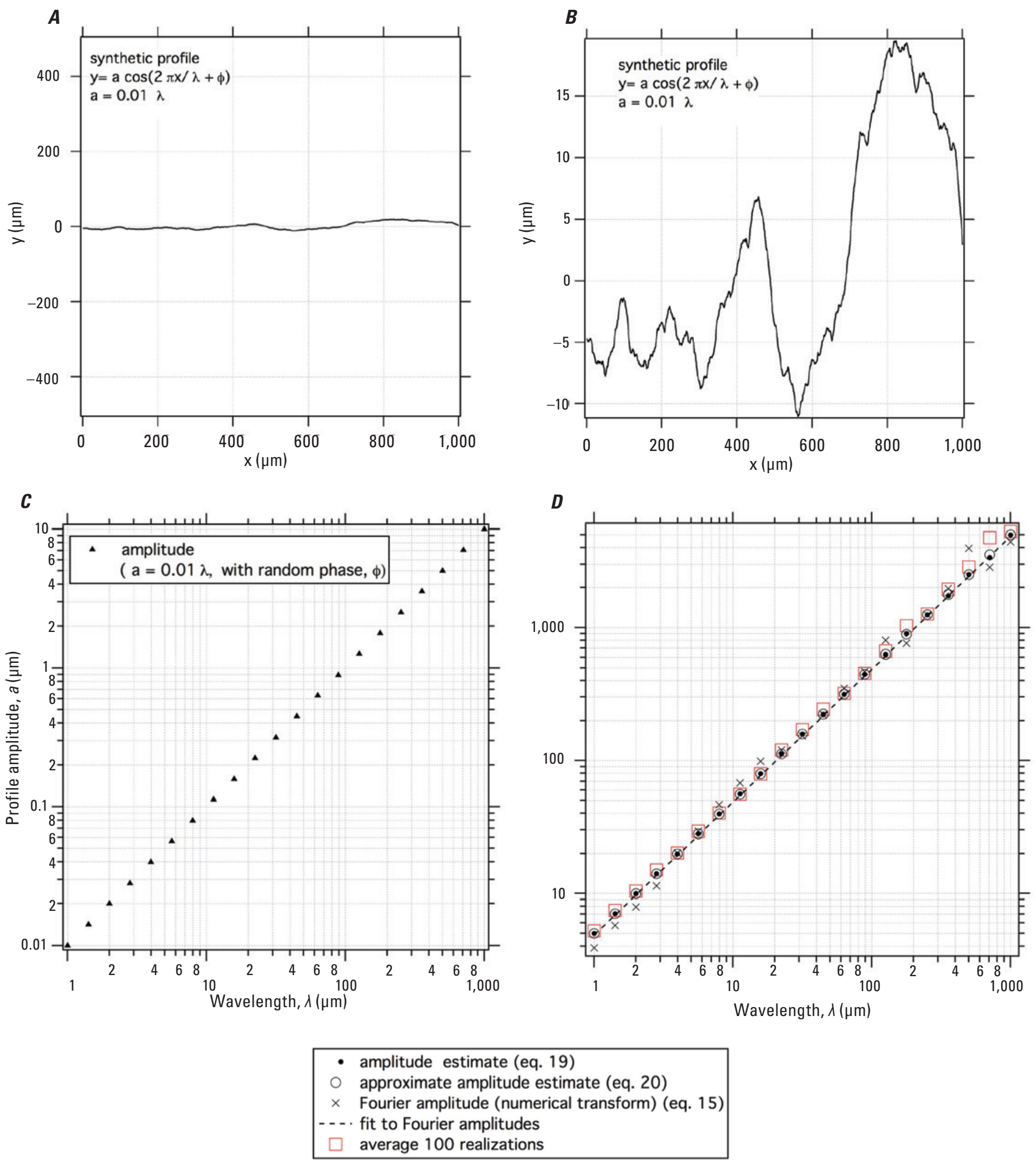

Figure 5. Simulated profile of length $L=1$ millimeter $(\mathrm{mm})$. Parts $A$ and $B$ (note scale difference) were constructed from summing 21 equally, logarithmically spaced cosine functions using equation 18 with $a=0.01 \lambda$. The assigned physical amplitudes are shown in part $C$. In $D$, the corresponding Fourier amplitudes, $A$, are estimated three different ways: with a numerical transform of the profile (eq. 15) at each of the 21 wavelengths, and using the analytical (eq. 19) and the approximate (eqs. 20 and 21) solutions of the specified parts. $a$, amplitude; $\lambda$, wavelength; $\mu \mathrm{m}$, micrometer; $\mu \mathrm{m}^{2}$, square micrometer. 
the constructed profile using the transform equation 15 . The estimated (eq. 20) and actual amplitudes (eq. 15) in this single realization are very similar. However, this is a single example with a specific set of random phases; in general, equations 18 , 19,20 , and 21 are only estimates. Collectively, they approximate the overall scaling (eq. 21) better than individual amplitudes at each of the chosen wavelengths. Included in figure $5 D$ in red symbols are the resulting amplitudes of the average for 100 different realizations of the construction (100 different sets of 21 random amplitudes), showing consistency but not identical values to the estimates. As the wavelengths approach $L_{p}$, the actual Fourier amplitudes begin to deviate notably from the estimates - this arises when the wavelength is not $\lambda / 4 \pi<<$ $L_{p}$ and therefore is not well resolved in equation 19. Note that the actual transform amplitude is also inconsistent with the assigned amplitudes in the construction at long wavelength. These are issues in interpreting fits to transforms of natural profiles at wavelengths near the profile length.

For representative profiles, since the $P_{\text {sd }}$ is the square of equation 18 normalized by profile length, $L_{p}$, the estimated dependence of $P_{\text {sd }}$ on wavelength relates to the dependence of the actual profile amplitudes on wavelength as

$$
P_{s d} \approx \frac{k_{\lambda}^{2} \lambda^{2 m} L_{p}}{4}
$$

Reemphasizing a prior result, the exponent $m$ that controls the amplitude scaling of the actual profile is half of $P_{\mathrm{sd}}$ and $m=\alpha / 2$. So, in addition to having a scale-independent ratio of average height to length $(\zeta=1 ; \alpha=3)$, equation 22 implies that a self-similar surface has a scale-dependent ratio of amplitude to wavelength, $m=3 / 2$. This requires that in the average sense, a self-similar surface has scale-independent roughness, but in the specific sense it does not.

The dependence of specific amplitude on wavelength is a requirement of self-similarity. That is, for the square root of the integral in the definition of $R_{\mathrm{q}}$ to be scale independent requires a specific continuous scale dependence of amplitudes. In detail, equating the RMS height with the average surface height (as done in the standard approach from Power and Tullis [1991]) in equation 2 and substituting in equation 22 results in

$$
\widehat{H}=R_{q}=\sqrt{\int_{1 / L_{p}}^{\infty} P_{s d} d f_{s}}=\sqrt{\int_{1 / L_{p}}^{\infty} \frac{d^{2} f_{s}^{-2 m} L}{4} d f_{s}}
$$

recalling that the spatial frequency $f_{\mathrm{s}}=1 / \lambda$. Carrying out the integration and evaluating results in

$$
\widehat{H}=\frac{d}{2} \sqrt{\frac{1}{2 m-1} L_{p}^{m-\frac{1}{2}}}
$$

For the ratio of average height to profile length to be independent of profile length $\left(\hat{H} \propto L_{p}\right)$ requires $m=3 / 2$, such that the specific amplitudes increase more rapidly with wavelength than wavelength itself.

This sense of the specific amplitude-to-wavelength scale dependence seems to conflict with the standard view of self-similar or natural fault surfaces. For example, for selfsimilar surfaces, $m$ exceeds 1 ; this means that the specific amplitude to wavelength ratio is higher at long wavelengths than it is at short wavelengths. As shown in figure 6, a selfsimilar trace is steeper than lines of constant $A / \lambda$. To a lesser degree, the same is true for natural fault surfaces, for which the examples shown in figure 4 are thought to be typical (Candela and others, 2015; Brodsky and others, 2015) and their slopes are slightly steeper than constant $A / \lambda$. These are reshown in figure 6 in the context of $A / \lambda$, where $m=1.1$ (Candela and others, 2015; Brodsky and others, 2015) and $m=1.3$ (Thom and others, 2017). Thus, in the specific sense, self-similar and natural fault surfaces are rougher at long wavelength than they are at short wavelength, in marked contrast to their profile-length-averaged properties.

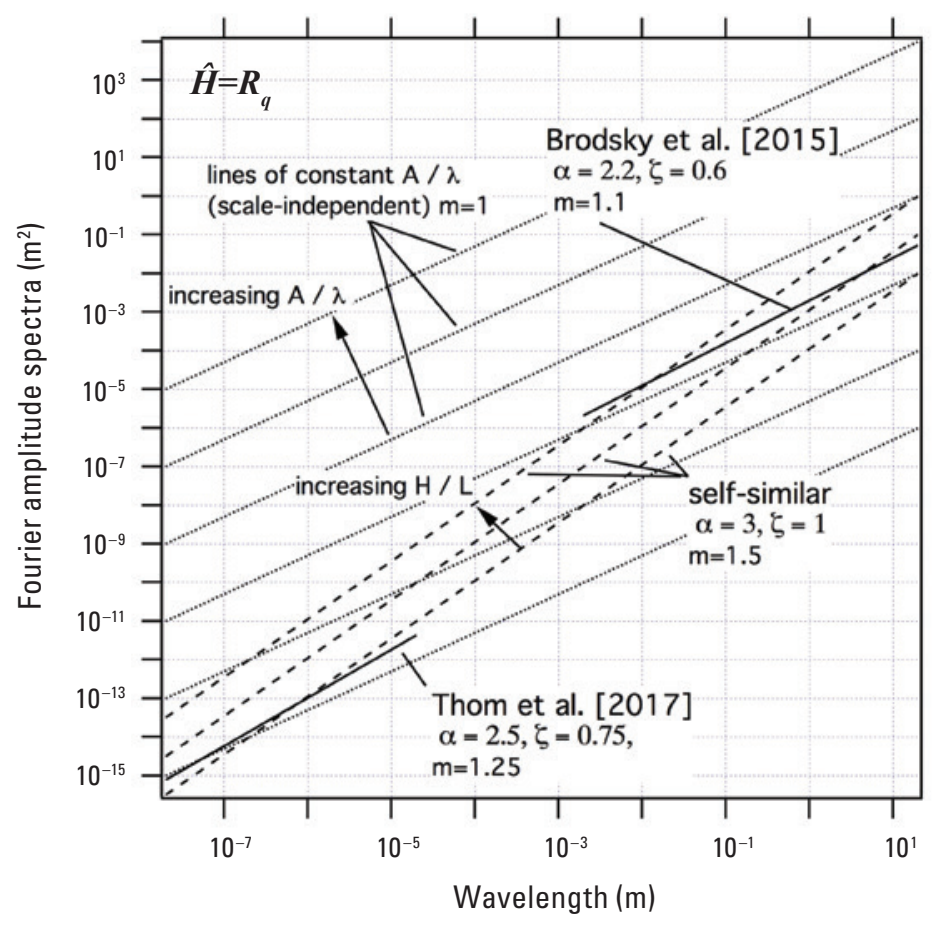

Figure 6. Summary Fourier amplitude plots for the assumption $\hat{H}=R_{q}$. Two natural fault surfaces are shown schematically in black (Brodsky and others, 2015; Thom and others, 2017). Here, since the trace lengths used to construct figure 4 are unknown, these are normalized assuming a length of 1 meter $(\mathrm{m})$ for each; their slopes are correct, but their locations are arbitrary. Example spectra of self-similar surfaces are shown as dotted lines of slope $m=3 / 2$, corresponding to constant average-height-to-profilelength ratios. Dotted lines are constant average-amplitude-tospecific-wavelength ratios with slope of $m=1 . \hat{H}$, average surface height; $R_{q}$, root mean square average roughness; $H$, surface height; $L$, surface length; $A$, fourier amplitude; $\lambda$, wavelength; $m$, scaling exponent in the physical realm; $\alpha$, power spectral density scaling exponent; $\zeta$, Hurst exponent; $\mu \mathrm{m}$, micrometer. 


\section{Discussion}

Generally, equations 20 and 21 emphasize that specific amplitudes of a rough fault surface can be approximately extracted from the transform normalized by half the profile length. Figure 7 shows a normalized transform, our Brown profile with the actual RMS height $(0.0022 \mu \mathrm{m}$, large open circle) plotted at the profile length. Having the profile amplitudes displayed in wavelength is conceptually useful for two reasons. First, amplitude at a particular wavelength is hard to visualize or measure in the physical domain except at long wavelengths. Average amplitude over a particular profile length from equation 2 is a more difficult concept. A normalized transform allows those to be reasonably well defined at all wavelengths. Second, for the unnormalized transform and for the standard power spectra approach, the resulting amplitudes depend on an arbitrary choice of profile length. Spectral amplitude from measurements made on the

\section{Synthetic random profile (brown noise)}

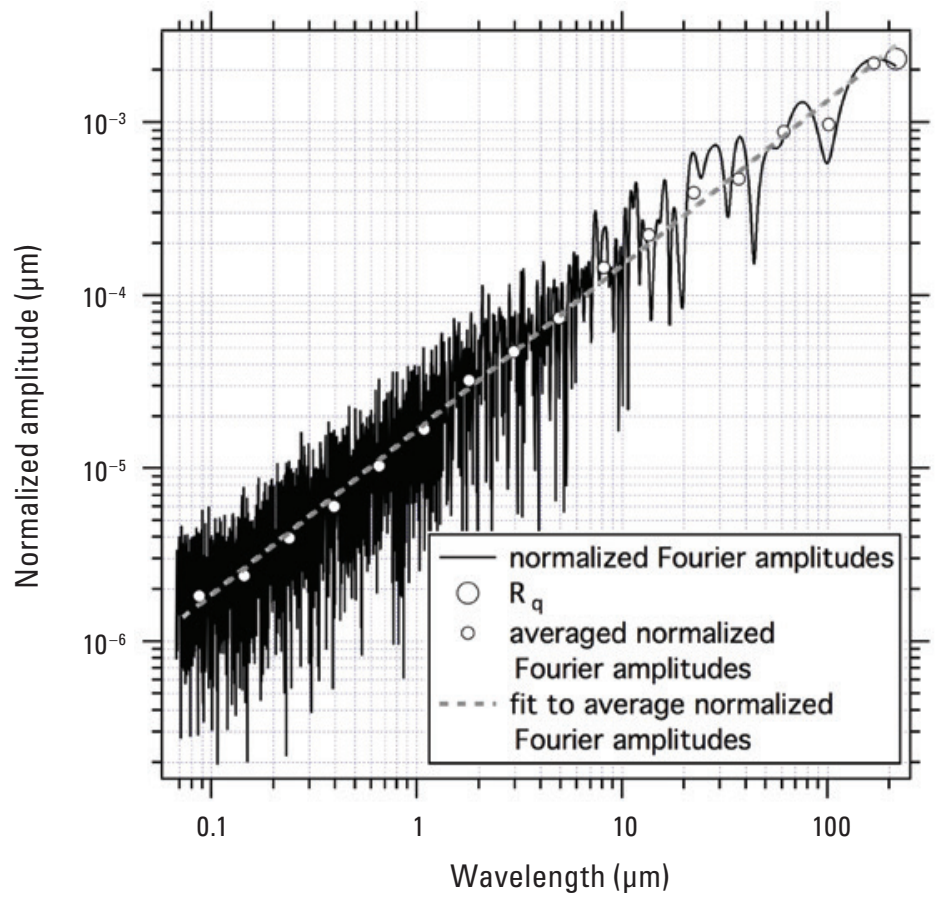

Figure 7. Plot of normalized Fourier amplitudes (in square micrometers $\left[\mathrm{mm}^{2}\right]$ ) versus wavelength (in micrometers [mm]). Amplitude spectra are normalized by half the profile length at 5,000 logarithmically spaced wavelengths. Shown for reference are the root mean squared average roughness $\left(R_{q}\right)$ calculated from the profile (large open circles), 16 logarithmically spaced average amplitudes (small open circles), and a fit to the average amplitudes, plotted in $\log (A)$ versus $\log (\lambda)$ space (dashed line). The fit values, calculated using equation 21 and normalized by half the profile length, are $k_{\lambda}=1.66 \times 10^{-5} \mathrm{~mm}, m=0.95 . R_{q^{\prime}}$ root mean square average roughness; $A$, Fourier amplitude; $\mu \mathrm{m}$, micrometers. same fault or from measurements of different faults aren't clearly tied to the actual roughness; this makes comparisons beyond the scaling exponent less straightforward. In addition to the scaling, presumably actual amplitudes and roughness resulting from fault creation and modification by shear reflect the underlying processes. For the normalized transform in figure 7, using equation 21, estimates of scaling exponent $m$ and the coefficient $k_{\lambda}$ (often called the "pre-factor" in the roughness scaling literature; for example, Renard and Candela [2017]) are available from a fit to the normalized amplitudes $A_{n}=k_{\lambda}\left(\lambda / \lambda_{0}\right)^{m}$. Here $\lambda_{0}$ is an arbitrary reference wavelength. Standard fast Fourier transform routines typically do not distribute amplitudes uniformly in wavelength; this can produce bias in any fit. In the example in figure 7 , the transform is calculated at Fourier amplitudes at 5,000 equally spaced logarithmic increments (lines). Those are then averaged across 16 equally (logarithmically) spaced bins (open circles), so that the fit (gray dashed line) is weighted uniformly across the range.

Table 2 summarizes the scaling relations with wavelength for average height, power spectral density, and actual surface amplitude for all the cases discussed in this report. Of note are the conditions where the profile length-averaged height and wavelength-average amplitude show opposite scaling, corresponding to Hurst exponents $0.5<\zeta<1$. These are profiles in the range between Brownian and self-similar. This is a curiosity of the statistics of rough surfaces that applies to nearly all natural fault surfaces.

Table 2. Summary of scaling exponents in descending degree of correlation (descending exponent) for different cases under the standard assumption that average surface height $(\hat{H})$ is the root mean square average roughness $\left(R_{q}\right)$.

[ $\zeta$ is the exponent in the scaling relation between average surface height and profile length, $\widehat{H} \propto L_{p}^{\zeta}$ (see eq. 2). $\alpha$ is the exponent in the scaling relation between power spectral density and wavelength, $P_{s d} \propto \lambda^{\mathrm{v}} \alpha$ (see eq. 8). $m$ is the exponent in the scaling relation between average amplitude and specific wavelength, $\hat{a} \propto \lambda^{m}$ (see eq. 3)]

\begin{tabular}{|c|c|c|c|}
\hline Case & $\begin{array}{c}\zeta=(\alpha-1) / 2 \\
\zeta=m-1 / 2\end{array}$ & $\begin{array}{c}\alpha=1+2 \zeta \\
\alpha=2 m\end{array}$ & $\begin{array}{c}m=\zeta+1 / 2 \\
m=\alpha / 2\end{array}$ \\
\hline Self-similar & 1 & 3 & $3 / 2$ \\
\hline $\begin{array}{l}\hat{H} \text { decreasing with } \\
\text { wavelength }\end{array}$ & $<1$ & $<3$ & $<3 / 2$ \\
\hline $\begin{array}{l}\text { Thom and others } \\
\text { (2017) }\end{array}$ & 0.75 & 2.5 & 1.25 \\
\hline $\begin{array}{l}\text { Candela and others } \\
\text { (2012); Brodsky } \\
\text { and others (2015) }\end{array}$ & 0.6 & 2.2 & 1.1 \\
\hline Brown noise & $1 / 2$ & 2 & 1 \\
\hline $\begin{array}{l}\hat{a} \text { decreasing with } \\
\text { wavelength }\end{array}$ & $<1 / 2$ & $<2$ & $<1$ \\
\hline Pink noise & 0 & 1 & $1 / 2$ \\
\hline
\end{tabular}


Are natural fault surfaces smoother at long wavelength? The answer clearly depends on the metric, which should be chosen based on the fault property of interest. In this regard, using additional roughness metrics may be useful in evaluating existing models of roughness genesis. For example, to explain the decrease in the ratio of average height to profile length with scale (fig. 4), Brodsky and others (2015) appeal to the scale-dependent intact strength of rocks and minerals, such as observed in laboratory failure experiments on samples of increasing specific size (Mogi, 1962; Pratt and others, 1971; see summary by Scholz, 1990). The standard interpretation of this scale-dependent strength is that failure occurs at a stress level that reflects a combination of the theoretical strength of the material and the number of flaws. Small samples have a smaller number of flaws and, in principal, can reflect the true theoretical material strength, whereas increasing sample size leads to progressively lower and lower values of failure strength (for example, Mogi, 1962). Using this scale-dependent strength to explain natural fault roughness only works if sample size in the experiments corresponds to profile length in an application to faulting and that size-dependent strength measured in the experiments corresponds to the size-dependent RMS strength of a rough fault. Foremost, of course, the argument requires that fault surfaces are smoother at long wavelength.

The motivation for exploring alternative roughness metrics and analysis procedures in this report is preliminary work to be applied to understand the evolution of natural and experimental fault surfaces during frictional sliding. For faults in laboratory experiments, our ultimate interests are in how roughness influences the degree of localization within the shear zone, absolute strength, and the rate dependence of fault slip. However, there are likely pitfalls to using spectral slopes alone to compare models to natural and laboratory data. For instance, the random walk profile has the same spectral slope (fig. $3 A$ ) as the example where amplitude varies linearly with wavelength (fig. 5). Their similarity in the amplitude spectral domain neither well reflects the notable differences in their synthetic genesis nor differences that are well defined in the physical domain. This is a common problem in interpreting amplitude spectrum representations without consideration of their series representation (for example, source models in seismology). Distinguishing between these two examples requires a broader view not used in standard roughness analyses. Some approaches would be to use phase information and additional roughness metrics, such as those developed in engineering studies of manufactured surfaces.

\section{Conclusions}

Prior studies of natural fault surfaces show that the ratio of surface average height to profile length decreases with scale, where average height is defined using the root mean squared roughness, $R_{\mathrm{q}}$. According to this approach, fault surfaces are smoother at long wavelength than they are at shorter wavelengths. However, a different roughness metric - the average heights that are specifically associated with a wavelength - shows the opposite sense of scaling. The ratio of amplitude to wavelength increases with wavelength. This apparent contradiction is a curiosity of the statistics of rough surfaces that have Hurst scaling exponents, $\zeta$, that relate profile length to $R_{\mathrm{q}}$ in the range of $0.5<\zeta<1$, as natural faults most commonly do. This expanded description of fault roughness is intended to provide usable constraints on physical mechanisms that generate and modify roughness during frictional sliding in laboratory experiments and the Earth.

\section{References Cited}

Amitrano, D., and Schmittbuhl, J., 2002, Fracture roughness and gouge distribution of a granite shear band: Journal of Geophysical Research, v. 107, p. 2,375-2,391, https://doi. org/10.1029/2002JB001761.

Barbour, A.J., and Parker, R.L., 2014, psd-Adaptive, sine multitaper power spectral density estimation for R: Computers and Geoscience, v. 63, p. 1-8, https://doi.org/10.1016/j. cageo.2013.09.015.

Brodsky, E.E., Gilchrist, J.J., Sagy, A., and Collettini, C., 2011, Faults smooth gradually as a function of slip: Earth and Planetary Science Letters, v. 302, p. 185-193, https:// doi.org/10.1029/2011JB009041.

Brodsky, E.E., Kirkpatrick, J.D., and Candela, T., 2015, Constraints from fault roughness on the scale-dependent strength of rocks: Geology, v. 44, p. 19-22, https://doi. org/10.1130/G37206.1.

Candela, T., Renard, F., Bouchon, M., Brouste, A., Marsan, D., Schmittbuhl, J., and Voisin, C., 2009, Characterizing fault roughness at various scales-Implications of threedimensional high resolution topography measurements: Pure and Applied Geophysics, v. 166, p. 1,817-1,851, https://doi.org/10.1007/s00024-009-0521-2.

Candela, T., Renard, F., Klinger, Y., Mair, K., Schmittbuhl, J., and Brodsky, E.E., 2012, Roughness of fault surfaces over nine decades of length scales: Journal of Geophysical Research, v. 117, no. B08409, https://doi. org/10.1029/2011JB009041.

Chatfield, C., 1975, The analysis of time series - Theory and practice: Springer, p. 263.

Chen, G., and Spetzler, H.A., 1993, Topographic characterization of laboratory-induced shear fractures: Pure Applied Geophysics, v. 140, p. 123-135.

Chester, F.M., and Chester, J. S., 2000, Stress and deformation along wavy frictional faults: Journal of Geophysical Research, v. 105, no. B10, p. 23,421-23,430, https://doi. org/10.1029/2000JB900241.

Dascher-Cousineau, K., Kirkpatrick, J.D., and Cooke, M.L., 2018, Smoothing of fault slip surfaces by scale-invariant wear: Journal of Geophysical Research, v. 123, no. 9, p. 7,913-7,930, https://doi.org/10.1029/2018JB015638. 
Davidesko, G., Sagy, A., and Hatzor, Y.H., 2014, Evolution of slip surface roughness through shear: Geophysical Research Letters, v. 41, p. 1,492-1,498, https://doi. org/10.1002/2013GL058913.

Dieterich, J.H. and Smith, D.E., 2009, Nonplanar faultsMechanics of slip and off-fault damage: Pure and Applied Geophysics, v. 166, p. 1,799-1,815.

Dunham, E.M., Belander, D., Lin, C., and Kozdon, J.E., 2011, Earthquake ruptures with strongly rate-weakening friction and off-fault plasticity, part 2-Rough faults: Bulletin of the Seismological Society of America, v. 101, p. 2,308-2,322.

Fang, Z., and Dunham, E., 2013, Additional shear resistance from fault roughness and stress levels on geometrically complex faults: Journal of Geophysical Research, v. 118, no. 7 , p. 3,642-3,654.

Gardner, M., 1978, Mathematical games-White and brown music, fractal curves and one over f fluctuations: Scientific American, v. 238, p. 16-33.

Kirkpatrick, J.D., and Brodsky, E.E., 2014, Slickenline orientations as a record of fault rock rheology: Earth and Planetary Science Letters, v. 408, p. 24-34.

Mandelbrot, B.B., 1967, How long is the coast of Britain? Statistical self-similarity and fractional dimension: Science, v. 156 , no. 3775 , p. $636-638$, https://doi.org/10.1126/science.156.3775.636.

Mandelbrot, B., and van Ness, J.W., 1968, Fractional Brownian motions, fractional noises and applications: Society for Industrial and Applied Mathematics, v. 10, no. 4, p. 422 437, https:/doi.org/10.1137/1010093.
Mogi, K., 1962, The influence of the dimensions of specimens on the fracture strength of rocks: Bulletin of the Earthquake Institute, v. 40, p. 175-185.

Power, W.L., and Tullis, T.E., 1991, Euclidean and fractal models for the description of rock surface roughness: Journal of Geophysical Research, v. 96, p. 415-424, https://doi. org/10.1029/90JB02107.

Power, W., Tullis, T., and Weeks, J., 1988, Roughness and wear during brittle faulting: Journal of Geophysical Research., v. 93, no. B12, p. 15,268-15,278, https://doi. org/10.1029/JB093iB12p15268.

Pratt, H.R., Black, A.D., Brown, W.S., and Brace, W.F., 1971, The effect of specimen size on the mechanical properties of unjointed diorite [abs.]: International Journal of Rock Mechanics and Mining Sciences and Geomechanics Abstracts, v. 9, no. 4, p. 513-516.

Renard, F., and Candela, T., 2017, Scaling of fault roughness and implications for earthquake mechanics, in Thomas, M.Y., Mitchell, T.M., and Bhat, S., eds., Fault zone dynamic processes - Evolution of fault properties during seismic rupture: Geophysical Monograph, v. 227, p. 197-215.

Schmittbuhl, J., Schmitt, F., and Scholz, C., 1995, The morphology of fractured rock surfaces: Journal of Geophysical Research, v. 100, p. 5,953-5,973.

Thom, C.A., Brodsky, E.E., Carpick, R.W., Pharr, G.M., and Oliver, W.C., 2017, Nanoscale roughness of natural fault surfaces controlled by scale-dependent yield strength: Geophysical Research Letters, v. 44, no. 18, p. 9,299-9,307, https://doi.org/10.1002/2017GL074663.

Voss, R.F., and Clarke, J., 1978, "1/f noise" in music—Music from 1/f noise: The Journal of Acoustical Society of America, v. 63, p. 258-263, https://doi.org/10.1121/1.381721. 


\section{Appendix. Voss-McCartney Algorithm for Pink Noise}

At every point along a discrete profile, the pink noise algorithm produces an amplitude that is the sum of $j+1$ random numbers, where $j$ is an integer. The total number of points in the profile is $N=2^{j+1}-1$, and for a profile length of $L_{p}$ the point spacing is $L_{p} /(N-1)$. All of the random numbers in the sum are updated at different periods such that, following J. McCartney (written commun., September 1999), there is an update at each point. To illustrate, a scalable example is shown in figure 1.1 where $j=3$, producing total points in the profile $N=15$, and $j+1=4$ random numbers. In this implementation, each generator selects numbers from a Gaussian distribution. The first step in the algorithm is to select initial values of all $j+1$ random numbers. Then, the amplitude at each point on the profile is determined sequentially by altering one of the $j+1$ values in the sum. Figure 1.1 shows the points in the profile horizontally from left to right and the random generator numbers from top to bottom. The first update positions and update periods are also shown, as follows.
The first update position for generators 1 to $j$ are at points $2^{j-i}$, where $i$ is the generator of interest. So, in the example shown in figure $1.1, j=3$ and for generator 1 the first update position is point number $4\left(=2^{3-1}\right)$. For generator 2 , the first update is at point number $2\left(=2^{3-2}\right)$, and so on. The single update position for generator 0 is at point $2^{j}$, point number 8 in the example. The first update positions for the four generators 0 to 3 are labeled along the top of grid in figure 1.1.

Similarly, for generators 1 to $j$ the update periods in number of points are $2^{j-i+1}$, where $i$ is the generator of interest. So, in the example, the update period for generator 3 is two points $\left(=2^{3-3+1}\right)$ and for generator 2 is four points $\left(=2^{3-2+1}\right)$. The update periods are labeled along the bottom of the grid in figure 1.1. Once the raw profile is produced from the procedure, the mean surface is removed by fitting a line to the raw synthetic and then subtracting the fit.

Distance along profile

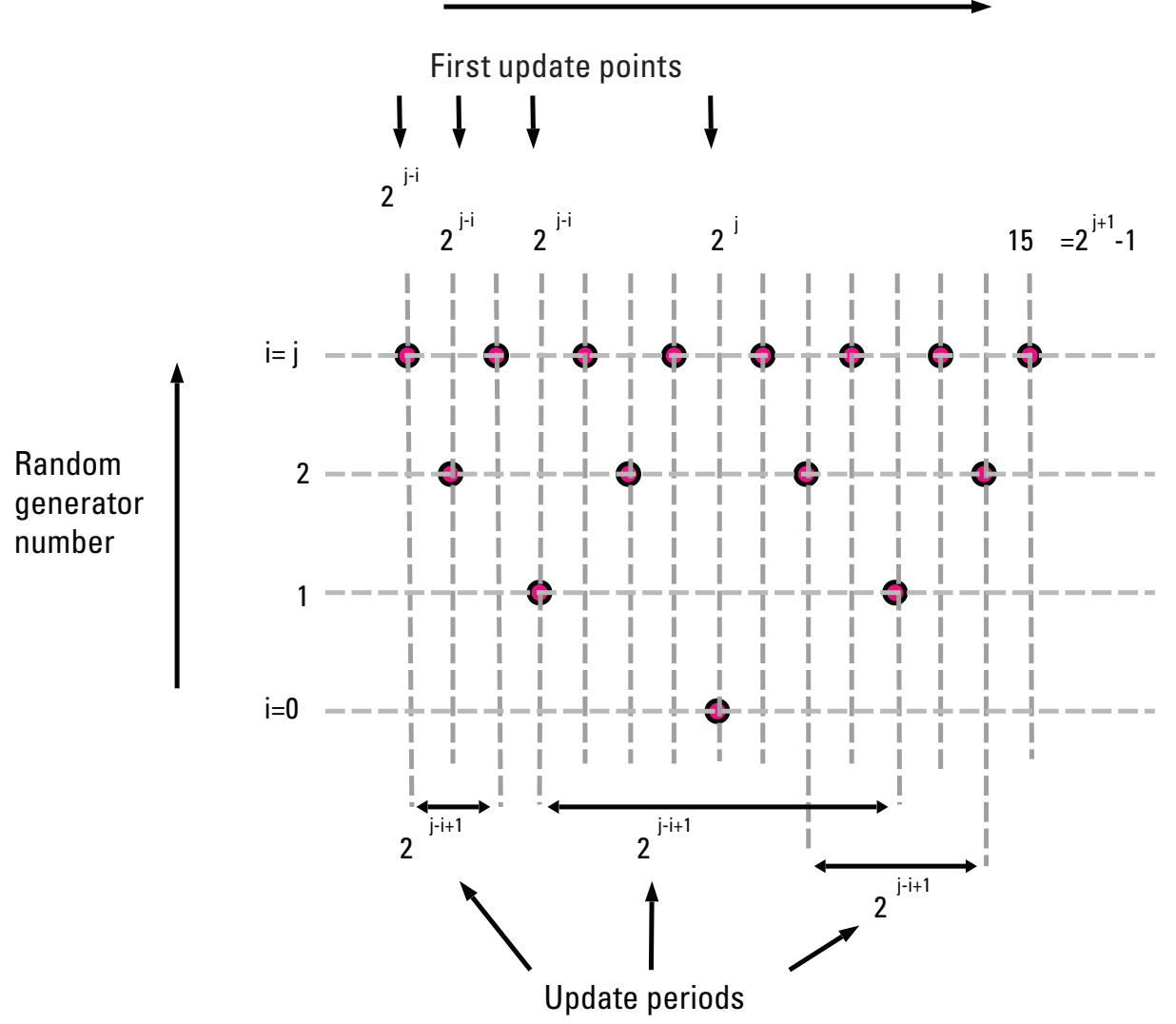

Figure 1.1 Schematic diagram showing the Voss-McCartney pink noise algorithm. Point position along the profile is shown horizontally at the top and the random generators are shown vertically. In this example, $j=3$. There are $N=2^{j+1}-1$ points (=15) and $j+1(=4)$ random number generators. The initial update position for generators 1 to $j$ are at points $2^{j-i}$, where $i$ is the generator number; their update period in number of points is $2^{j-i+1}$. The single update position for generator 0 is $2^{j}$. The update points for each generator are labeled along the top of the grid and the update periods are labeled along the bottom. 
Moffett Field Publishing Service Center, California

Manuscript approved for publication November 20, 2020

Edited by Aditya Navale and Monica Erdman

Layout and design by Kimber Petersen 
